\title{
Experiencia de los cuidadores informales en el manejo de la diabetes mellitus tipo II
}

\author{
Caregivers' experience on dealing with diabetes mellitus type II
}

Experiência na gestão dos cuidadores informais da diabetes mellitus tipo II

\author{
María L. Pinzón-Rocha ${ }^{1 *}$, Luz H. Aponte-Garzón ${ }^{2 *}$, Ruth F. Hernández-Páez ${ }^{3 *}$

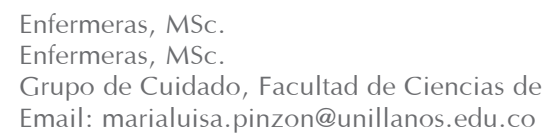

Recibido: Noviembre 9 de $2012 \quad$ Aceptado: Junio 7 de 2013.

\begin{abstract}
Resumen
Las personas con diabetes representan una carga significativa tanto para los servicios de salud, como para las personas que ofrecen cuidado informal en los domicilios. En la actualidad se reconoce el cuidado de los cuidadores informales como un elemento importante para la obtención y mantenimiento del control metabólico de las personas con diabetes. El propósito de este estudio fue explorar la experiencia vivida por cuidadores informales en el manejo de la Diabetes Mellitus tipo 2. Ello permitió conocer realidades de convivencia con la enfermedad, descubrir significados y perspectivas de los cuidadores en el desempeño de su rol, para mantener en las mejores condiciones de salud a las personas que padecen esta enfermedad. El estudio fue fenomenológico, con entrevistas a profundidad para establecer narraciones del fenómeno de interés e identificar elementos significativos de la perspectiva personal del cuidado cotidiano por parte del cuidador informal. La muestra fue obtenida por conveniencia y estuvo conformada por 15 cuidadores informales residentes en el municipio de Villavicencio-Meta. La información se analizó siguiendo la metodología de Colaizzi para la determinación de categorías de análisis relacionadas con la comprensión de la enfermedad, prácticas de cuidado cotidiano, percepción de la enfermedad y necesidades de apoyo familiar y social. Cada una de estas categorías son representativas de la experiencia cotidiana de cuidar a personas con esta patología. Los resultados señalan que convivir y manejar la situación de Diabetes es una realidad compleja para la mayoría de los cuidadores estudiados, debido a la falta de comprensión sobre el desarrollo de la enfermedad, al cuidado cotidiano mediado por prácticas del saber científico y popular, a sentimientos de angustia e incertidumbre por la variabilidad y difícil manejo de la enfermedad, y por la falta de apoyo familiar y social para adoptar y mantener cambios en el estilo de vida.
\end{abstract}

Palabras claves: Cuidadores, Diabetes Mellitus, Educación en salud.

\begin{abstract}
People with diabetes represent a significant burden to health systems as well as to the informal caregivers at the domiciliary level. At the present time care offered by informal caregivers is recognized as an important element for obtaining and maintaining metabolic control in diabetic patients. The purpose of this study was to
\end{abstract}


explore the experience lived by informal caretakers in dealing with Type 2 Diabetes, which allowed knowing realities of coexistence with the illness, to discover meanings and caregivers' perspectives in their role in order to maintain the best possible health conditions of people with this disease. The study was phenomenological, with in depth interviews to establish expressions of the phenomenon of interest and to identify significant elements of their perspectives about everyday care. A convenience sample was used $(n=15)$ conformed by informal caregivers residents in Villavicencio, Meta. Information was analyzed according to Colaizzi method, and analysis categories were determined related to understanding of the illness, practical of daily care, perception of the illness and necessities of family and social support. Each one of these categories represents the daily experience of caring for people with type 2 diabetes. Results showed cohabiting and managing the diabetes situation is a complex reality in most of the studied caregivers due to the lack of understanding of the disease, to the care guided by scientific and popular knowledge, feelings of anguish and uncertainty due to disease variability and difficulty in its management, and lack of family and social support to adopt and maintain lifestyle changes.

Key words: Caregivers, Diabetes mellitus, Health education.

\section{Resumo}

As pessoas com diabetes representam um ônus significativo para ambos os serviços de saúde para as pessoas que prestam cuidados informais nas casas. Atualmente se reconhece a função dos cuidadores informais como um elemento importante para obtenção e manutenção do controle metabólico de pessoas com diabetes. O objetivo deste estudo foi explorar a experiência vivida pelos cuidadores informais na gestão do diabetes mellitus tipo 2, o que permitiu conhecer a realidade de viver com a doença, descobrir significados e perspectivas dos profissionais de saúde na realização de seu papel, para manter em melhores condições de saúde as pessoas com esta doença. O desenho do estudo foi fenomenologico, com entrevistas em profundidade para estabelecer narrativas do fenômeno de interesse e identificar elementos significativos do ponto de vista pessoal de cuidados diários pelo cuidador informal. A amostra foi obtida por conveniência e consistiu em 15 cuidadores informais que vivem na cidade de Villavicencio, Meta. Os dados foram analisados seguindo a metodologia do Colaizzi para determinar categorias de análise relacionadas com a compreensão da doença, as práticas de cuidado diário, a percepção da doença e as necessidades de apoio familiar e social, cada um deles representante da experiência diária para cuidar de pessoas com essa situação de doença. Os resultados para viver e lidar com a Diabetes situação é uma realidade complexa, na maioria dos cuidadores estudados, devido à falta de entendimento sobre o desenvolvimento da doença, as práticas de cuidado diário mediadas pelo saber científico e popular, para os sentimentos ansiedade e incerteza e a variabilidade difícil manejo da doença e da falta de apoio familiar e social para adotar e manter mudanças no estilo de vida de suporte.

Palavras-chave: Cuidadores, Diabetes Mellitus, Educação em saúde.

\section{Introducción}

Una de las enfermedades que ha contribuido de manera significativa a la presencia creciente de enfermedades crónicas y el deterioro progresivo de la salud de la población en los diferentes contextos internacional, nacional y regional, es la Diabetes Mellitus tipo 2 (DM 2). Se caracteriza por una elevada concentración plasmática de glucosa que genera alteración en el metabolismo de proteínas, grasa y carbohidratos. Se trata de un síndrome complejo con repercusiones agudas y crónicas principalmente a nivel vascular, que ocasiona complicaciones irreversibles y muerte de las personas que la padecen a mediano y largo plazo. Actualmente la DM 2 es considerada un problema de salud pú- blica con enormes repercusiones sociales, generadora de alta morbilidad y mortalidad, particularmente en las sociedades desarrolladas o en vías de desarrollo (Sociedad Andaluza de medicina familiar y comunitaria, 2008). Organismos internacionales predicen un aumento sostenido de casos. Para el año 2030 habrá cerca de 339 millones de personas con diabetes. En principio, los factores asociados a este incremento son la obesidad, el sedentarismo, la urbanización de la población y la transición demográfica (Federación Internacional de Diabetes, 2012).

Se reconoce que la DM 2 es una de las causantes principales de invalidez y de pérdida de años de vida saludable a causa de las complicaciones sistémicas que 
genera. El coma hiperosmolar, la cetoacidosis diabética, ceguera, enfermedad renal, ataques cardiacos y amputaciones, entre otras. La vivencia de esta situación durante un periodo largo de vida tiene un gran impacto a nivel personal, familiar e institucional debido a los cambios en el estilo de vida que se hacen más difíciles en la edad adulta y al alto costo económico para el control y prevención de las posibles complicaciones (Federación Internacional de Diabetes, 2012).

El manejo de esta situación patológica es más de cuidado preventivo que curativo. La responsabilidad del cuidado recae principalmente en la persona que la padece y en los miembros de la familia. Por lo general la labor de cuidar se le delega a una persona que se compromete a realizar las acciones del caso, de forma permanente y durante un tiempo prolongado. Se les llama cuidadores principales o informales. Ellos constituyen la principal forma de brindar cuidado informal y domiciliario a nivel mundial (Carretero, et al., 2006).

Los cuidadores informales son un componente esencial del cuidado crónico, debido a que tienen una gran responsabilidad en la satisfacción de necesidades de cuidado, procesos adaptativos frente a la enfermedad y respuestas positivas en adherencia a tratamientos. Indagar por la experiencia, sentimientos y significados que tienen los cuidadores acerca de esta situación de salud, permite analizar e interpretar el desempeño de su rol, así mismo establecer mecanismos que permitan mejorar su habilidad de cuidado (Astudillo et al., 2008).

En este sentido, el grupo investigador se propuso conocer la experiencia del cuidado vivida por los cuidadores informales de la región en el manejo de la DM 2 , teniendo en cuenta que el éxito de manejar adecuadamente la situación de salud, parte del conocimiento, la comprensión y el cuidado diario que se brinde a la persona que padece la enfermedad. Aprender a convivir y manejar ésta situación crónica, es una herramienta fundamental para mantener el control metabólico y evitar la aparición de complicaciones, contribuyendo así a fortalecer el autocuidado, el bienestar y la calidad de vida de las personas y familias en general (Bustos et al., 2007).

\section{Materiales y métodos}

Estudio descriptivo de abordaje fenomenológico en el marco de la investigación cualitativa, que permitió indagar y describir la experiencia humana de los cuidadores informales con respecto al manejo cotidiano de la DM 2. La descripción de la experiencia se rea- lizó con 15 cuidadores informales seleccionados por conveniencia, que cumplieron con los criterios de participación establecidos en el estudio: ser cuidador mayor de 18 años, haber cuidado a una persona con diagnóstico de DM 2 por lo menos durante un año, ser residente de la ciudad de Villavicencio y aceptar voluntariamente la participación en el estudio.

La recolección de la información se hizo a través de entrevista a profundidad, en el domicilio de los cuidadores, previo consentimiento informado. La duración aproximada de la entrevista fue entre una a dos horas, utilizando una a dos sesiones. La indagación se inició preguntando sobre el tiempo que lleva como cuidador de una persona con DM 2, qué conoce sobre la enfermedad y cómo ha sido la experiencia de cuidado. Las entrevistas fueron grabadas y transcritas literalmente para su correspondiente análisis.

La información se analizó siguiendo la metodología propuesta por Colaizzi 1978 (Shosha, 2012), la cual establece los siguientes pasos: 1) Recolección de la información de los participantes sobre el fenómeno previamente definido. 2) Lectura de todas las transcripciones de las entrevistas. 3) Determinación de enunciados significativos. 4) Definición de categorías de análisis a partir de los enunciados significativos. 5) Descripción teórica de las categorías de análisis 6) Descripción del fenómeno a la luz de las categorías de análisis. 7) Triangulación de datos para la validación de la descripción. 8) Incorporación de nuevos datos a la descripción del fenómeno si fuese necesario, con el fin de enriquecer la construcción de significados y resultados de la investigación (Sánchez, 2000).

Teniendo en cuenta los criterios establecidos en el Artículo 6 de la Resolución 08430 de 1993 del Ministerio de Salud, el trabajo no presentó riesgos para las personas ni para el ambiente. Se mantuvo el rigor ético frente a la seguridad e integridad de los participantes, consentimiento informado, recursos materiales necesarios y participación de personal de enfermería idóneo, con el fin de garantizar el bienestar de los participantes en la investigación. Con el propósito de mantener la confidencialidad de la identidad de los participantes, a cada uno de ellos se le asignó un código que fue usado en las transcripciones y en la selección de enunciados significativos correspondientes.

\section{Resultados y discusión}

Los cuidadores se caracterizaron por ser, en su mayoría, mujeres pertenecientes a los estratos socioeconómicos 1, 2 y 3, que asistían a los servicios de crónicos 
de la Empresa Social del Estado (ESE), con niveles educativos de primaria y secundaria incompleta. Todos tenían relación familiar principalmente en el cuidado de padres y esposos, con un tiempo promedio de 12 años de enfermedad con presencia de algún grado de complicación como Insuficiencia Renal, coma diabético, pie diabético y secuelas de ACV. Los cuidadores convivían en el mismo domicilio con los sujetos de cuidado.

El análisis de la experiencia de los cuidadores informales en el manejo de la DM 2 permitió establecer cuatro categorías de análisis relacionadas con la comprensión de la enfermedad: prácticas de cuidado cotidiano, percepción de la enfermedad y necesidades de apoyo familiar y social. Cada una de ellas son representativas de la experiencia cotidiana de cuidar a personas con esta patología. A continuación se presenta la descripción y análisis de cada una de las categorías establecidas.

\section{Comprensión de la Enfermedad por parte del cuidador informal}

Teniendo en cuenta la complejidad metabólica de la DM 2 y su incidencia en la calidad de vida de las comunidades a nivel mundial, el conocimiento y comprensión que tienen las personas que la padecen y cuidan de ella, cobra fundamental importancia frente al logro del bienestar y la calidad de vida de individuos y familias. Algunos estudios (Noda, 2008; Rodríguez et al., 2009) señalan que hay una clara relación entre los conocimientos insuficientes para el manejo de la enfermedad y la aparición de complicaciones. Así mismo, reportan que los pacientes y cuidadores pueden conocer el nombre de la enfermedad pero no saben en qué consiste, ni los factores de riesgo que pueden contribuir a descompensarla.

A continuación se resaltan algunos testimonios que muestran la comprensión que tienen los cuidadores informales sobre la enfermedad que manejan:

C1: Lo que yo conozco de esta enfermedad es que hace daño en varias partes del cuerpo, que se debe tener un control extremo en las comidas, que la persona que la tiene puede enfermarse de muchas otras cosas más, de las complicaciones, no se mucho.

E1: Hasta donde yo tengo conocimiento, es una enfermedad como dicen lenta, silenciosa, eso es como el que tiene cáncer, si no se cuida, es una enfermedad que le destruye hasta los órganos.
G4: Tengo entendido que va acabando con los órganos de uno, como la vista.

13: "Solo sé que es una enfermedad de cuidar... me han dicho que es una enfermedad delicada, no sé qué tipo de diabetes será, solo sé que hay que cuidarlo a él."

K4: "Yo entiendo que la Diabetes es como un virus o como un bichito que daña los riñones, el corazón y el hígado... hagamos de cuenta que eso es como un cáncer que hecha a dañar todo lo de adentro de uno..."

K5: "No sé por qué le dio Diabetes, eso es una enfermedad que no se sabe en qué momento le da a uno..."

N15: "...yo miraba a mi mamá y decía mi mamá no tiene diabetes, son mentiras, porque yo la miro de buen color, yo la miro que ella come bien"

L3: "No sé nada de la Diabetes, pero tengo la experiencia de cuidarla a ella"

Las expresiones de los cuidadores informales destacan una comprensión relativamente escasa de la enfermedad. Sin embargo consideran que su manejo es importante para que no se presenten complicaciones que pueden ir desde un grado menor hasta otro de gravedad considerable. El conocimiento insuficiente de la enfermedad y de sus formas de manejo por parte de los cuidadores informales, se traduce, por un lado, en alto riesgo de deterioro y de complicación para quien la padece. De otro lado, en una oportunidad para el apoyo educativo por parte de los profesionales de la salud. Los de Enfermería en particular, quienes deben cumplir un rol protagónico en el acompañamiento de la persona con DM 2 y su familia para el control de la enfermedad y la prevención de complicaciones (Rodríguez et al., 2009).

Expresiones como "no sé nada", "No sé porque le dio", "solo sé que es una enfermedad de cuidar", "Sé que es como un cáncer que destruye órganos" y "solo sé que hace daño en varias partes del cuerpo", determinan el escaso conocimiento de la etiología del padecimiento, lo cual aumenta la probabilidad de un manejo inadecuado por parte del cuidador por desconocimiento y falta de comprensión de la enfermedad. Estudios adicionales (Venegas, 2006; Vega et al., 2009) han referido que la habilidad del cuidador y la funcionalidad de la persona cuidada, depende en gran medida del grado de conocimiento que se tenga de la enfermedad, el acompañamiento y el apoyo cognitivo que se le pueda 
brindar al cuidador. Así mismo (Millar, 2008) reporta que el nivel de conocimiento sobre diabetes entre pacientes y cuidadores, generalmente es insuficiente. Lo anterior sugiere una educación y acompañamiento deficiente por parte del equipo se salud hacia las personas involucradas en el cuidado de esta enfermedad. La orientación y apoyo familiar son fundamentales para el logro de las metas que tienen que ver con el control metabólico prevención de complicaciones.

La literatura establece que la familia es, en primera instancia, la responsable de brindar cuidados y atender las necesidades que desencadena el curso de la enfermedad crónica. Enfrentar esta situación requiere de acompañamiento, formación y supervisión, con el fin de fortalecer el conocimiento y el desarrollo de habilidades para apoyar el manejo adecuado de la enfermedad, favorecer la adaptación y la funcionalidad de familias y cuidadores informales ante los requerimientos de la DM 2 (Sánchez et al, 2008; Grau et al., 2010). Otros estudios plantean que fortalecer el conocimiento sobre el cuidado en situaciones crónicas, además de garantizar un manejo adecuado de la enfermedad, disminuye los altos costos de la estancia hospitalaria de los pacientes y el estrés de todos los involucrados, generado por la incapacidad de afrontar situaciones desconocidas a causa de la enfermedad. (Martinez et al., 2006; Zavala et al., 2009).

Particularmente esta patología, asociada a estilos de vida poco saludables, obesidad y sedentarismo, requiere de adecuada orientación, comprensión de la situación, habilidades en los cuidados y motivación para asumir la responsabilidad del cuidado y responder de manera efectiva y eficaz a los retos que impone cada día la enfermedad (Pérez et al., 2009). Responder a esta problemática es responsabilidad de los profesionales de enfermería, quienes deben fortalecer la educación en salud a enfermos y cuidadores sobre lo cuidados y los recursos personales para el empoderamiento en el manejo de las circunstancias difíciles que podrían enfrentar en el curso de la enfermedad. De igual forma, la literatura plantea que existen otros factores asociados como las actitudes positivas, el valor, la paciencia, la confianza y la motivación por el aprendizaje, los cuales reducen el estrés asociado a la enfermedad, aumentan la disposición para asumir el cuidado y mejoran la eficacia de los mismos (Ortiz, 2011). Tanto el conocimiento de la enfermedad crónica, como el conocimiento de ser cuidador, se constituyen en herramienta indispensable para brindar un mejor cuidado. Cuando no se cuenta con estos elementos, la habilidad del cuidado puede estar deficientemente calificada y disminuye la probabilidad de tomar decisiones asertivas (Gonzalez, 2006).
Como toda enfermedad crónica, esta situación requiere de un mayor cuidado, de tal forma que el componente de apoyo educativo, como lo plantea Orem (Raile, 2011), es una responsabilidad que debe asumir el equipo de salud. El propósito de lo anterior es garantizar que pacientes y familias comprendan la magnitud del trastorno metabólico y las posibles complicaciones de la DM 2. De la misma forma asuman el compromiso de realizar intervenciones de autocuidado que faciliten un óptimo control y manejo adecuado de la enfermedad.

\section{Prácticas de cuidado cotidiano de los cuidadores informales}

Es evidente que las prácticas de cuidado forman parte de la cotidianidad de las personas para proteger $y$ preservar la vida. Así mismo se constituyen en expresiones del acervo cultural que caracteriza la forma de pensar y de actuar con respecto a la salud y su cuidado (Morrison, 2007). Las guías de atención de la DM 2, nacionales e internacionales, establecen que el cuidado de esta enfermedad debe estar orientado a un manejo que integre varios aspectos como: el control de glicemia y presión arterial, ingesta de una alimentación saludable, control del peso corporal, incremento de la actividad física, toma de medicamentos, suspensión del hábito de fumar, cuidado de los pies, control emocional y un óptimo nivel educativo sobre la naturaleza y desarrollo de la enfermedad. Adicionalmente, recomiendan que las intervenciones sean acordadas entre paciente, familia y equipo de salud, evaluando parámetros culturales para ofrecer la mejor calidad de vida posible (UDA, Guía 17 de atención a la Diabetes; Asociación Latinoamericana de Diabetes, 2006).

El análisis de las prácticas de cuidado hechas por los cuidadores, permitió observar la disposición e interés por el cuidado y manejo de la enfermedad, todos orientados principalmente en el manejo de la alimentación con restricción de azucares y harinas. Sin embargo, en la práctica, esta intención puede verse interferida cuando no existe una clara comprensión de los alimentos que las contienen. La experiencia de los cuidadores evidencia que no cuentan con el conocimiento, ni con los recursos suficientes para lograr el alcance del nivel óptimo de glicemia. Otros cuidados como la realización de ejercicio, el control de la glicemia, el cuidado de los pies y el control emocional entre otros, no fueron mencionados por lo cuidadores. Los siguientes enunciados muestran la cotidianidad de las prácticas de cuidado.

D1: No le damos harinas, solo sopa de verduras, frutas no, eso nos han puesto, una dieta muy "verraca", y esa dieta uno casi no se la puede ayudar a llevar. 
I12 "Yo lo cuido a mi manera, no todos los días tengo la oportunidad de comprarle lo que necesita; la mazamorra se la come simple y todos los jugos también, al desayuno a veces es arepa de promasa, unas galletas integrales con aguapanela bajita de dulce."

F1: Primeramente dejarle la comida sin sal, y que bastante verdura, no darle grasa, no darle dulce, todo bajito de sal y sin dulce, la droga pues hay que dársela conforme al médico se la explica a uno. El tratamiento que le dan yo lo realizo conforme me dicen las formulas.

G6: Se manejan menos harinas, más verduras, no comer en exageración, sino comer poquito y fraccionado.

H7: Cuando uno está bien puede consumir lo normal, pero al sufrir una diabetes tiene que cambiar todo: los friticos, sus arepitas, sus pancitos y su leche.

116: Ahí le tengo la insulina pero como no le tengo la jeringuita pues no se la estoy colocando, pero se está tomando el alpiste desde que salió del hospital.

G7: Se ha dado el caso de usar yerbas medicinales que me han recomendado para cuando está muy elevada del azúcar, yo uso una que le dicen garrapata y la consigue en cualquier borde de anden y de verdad que es muy buena, eso le ha ayudado muchísimo.

H10: Uno tiene que ayudarse con remedios caseros, él toma mucha agua pura y agua con limón, pero cuando la gastritis no lo permite, toma la insulina en hojas, o consume la alcachofa que también dicen que es bueno.

I15: Ahorita está tomando alpiste de ese de los loros y dice que se siente relajado, que se siente bien, que no le ha vuelto a dar fiebre.

K10: "Le preparo bebidas con hierbas, algunas son buenas para manejar la diabetes por ejemplo esa que Ilaman casco de vaca, un pocilladito de esos es muy bendito"

Estas prácticas cotidianas de cuidado, se enmarcan en lo planteado por otros estudios (Luengo et al., 2010; Sapag et al., 2010; Vaquiro et al., 2010), que muestran cómo esta situación crónica tan compleja, es asumida por cuidadores informales con mínima información para tomar decisiones, evaluar la situación de salud y mantener el control metabólico de la enfermedad. Éste es complejo y está condicionado por factores socioculturales, psicológicos y educacionales que requieren de apoyo individual y familiar para mejorar la eficacia de los cuidados y lograr una mejor adaptación a los cambios que requiere la enfermedad (Universidad de Azuay, 2009).

Las actuaciones de los cuidadores dejan ver el desarrollo de prácticas relacionadas con el saber popular enmarcadas dentro del ámbito de la medicina alternativa. Este aspecto está estrechamente ligado con el contexto sociocultural de los cuidadores informales pertenecientes al municipio de Villavicencio, región que hace parte de la Orinoquia Colombiana. Ella está conformada socialmente por indígenas y colonos con problemas sociales similares a otras regiones del territorio nacional, como el conflicto armado, procesos migratorios forzados, pobreza e inequidad, necesidades básicas no satisfechas, violencia y baja cobertura de los servicios de salud entre otras condiciones, que crean alta vulnerabilidad e incertidumbre para la supervivencia y desarrollo (Sarmiento et al., 2010; Torres et al., 2009).

Estudios sobre la cultura de los cuidados establece que las situaciones del contexto que conllevan a las personas a vivir realidades de pobreza, marginación, exclusión y falta de apoyo social entre otras, permiten visibilizar con mayor frecuencia prácticas de cuidados relacionadas con sus creencias y costumbres trasmitidas a través de generaciones, con el propósito de satisfacer necesidades y mejorar sus condiciones de vida (Romero, 2009). En este marco, el reconocimiento de experiencias de cuidado alternas a la medicina convencional, no pueden ser ajenas al profesional de enfermería, su valoración y validación permitirán determinar el aporte al cuidado de la salud y la posibilidad de ser involucrado en la práctica profesional (Castillo, 2008). Explorar los aspectos cualitativos del cuidado permite la valoración de los patrones culturales específicos que, una vez reconocidos, podrán ser utilizados en beneficio de las comunidades. Así mismo, se ha establecido que tanto los saberes populares como los científicos tienen un contexto histórico y dialéctico, que incide de manera directa en el cuidado de la salud. Por lo mismo, ameritan su articulación a través de métodos hermenéuticos que faciliten el análisis y las interpretaciones de las diferentes acciones humanas con respecto al cuidado de la salud (Siles, 2010).

\section{Percepción de la Enfermedad por parte del cuidador informal}

La enfermedad vista como un estado de alteración de la salud es un predictor significativo de la actitud y conductas hacia el cuidado en reacción a los síntomas, 
evolución y expectativas de la enfermedad (Medina et al, 2009). Las creencias, percepciones y actuaciones en el cuidado de la salud, están determinadas por patrones socioculturales, motivación o interés por la salud y por el momento histórico en el que se presenta una determinada situación (Quiceno et al., 2010).

En este caso la DM 2 es percibida por los cuidadores con incertidumbre, preocupación e incapacidad por el comportamiento variable que ella encierra con la presencia ocasional de etapas críticas. Así, la enfermedad es percibida de difícil manejo, debido a que se deben atender diversos aspectos de la persona enferma, tanto en su condición física como emocional. Para la mayoría de cuidadores, el diagnóstico de la enfermedad y el desempeño del rol fueron inesperados, situación que afectó de manera significativa a la persona enferma y su familia. Consideran que el deterioro progresivo del sujeto de cuidado les produce tristeza y desesperanza, aceptan su rol pacientemente y su fortaleza se la atribuyen al acompañamiento de Dios, ser supremo.

La percepción de la enfermedad como condición que puede generar estados graves de la condición de salud, hace que los cuidadores se interroguen frecuentemente sobre qué van a hacer. Sienten que desconocen mucho sobre el cuidado que deben aportar al familiar y se sienten inseguros frente al desempeño de su rol. Algunas expresiones sobre esta categoría se presentan a continuación:

A2: Yo lo veo como un factor de riesgo inmenso, eso me da mucho miedo. La experiencia con mi abuelo, no quiero que también se dé con mi mami. Es una preocupación muy grande, eso me pone triste.

B1: Hay muchos cambios, porque yo vivo con esa incertidumbre, de que él se me sienta mal de algo, yo digo que es una complicación ya de eso.

C1: Cuando me acuerdo de eso me da mucha tristeza, porque a mi hermana, pues la quiero y uno nunca quisiera algo malo para la familia.

C2: La tristeza esta todos los días, pero la motivación para mí y para ella son esas dos chinas pilas que tiene por hijas, y yo se que por ellas dos ella sigue viva.

D1: Ella ya no vive tranquila, sino deseando la muerte, eso es como el pensado de ella, entonces, por eso ha cambiado, porque ella vive deseando morirse, por eso vive como amargada, si, ella tampoco no se puede controlar.
E1: Uno al comienzo se siente, que es incapaz, porque lo que yo he bregado con él ha sido mucho, porque él ha tenido unos comas graves. Uno se preocupa bastante, porque imagínese eso se inflama, le da mucha sed, fiebre, no le baja el azúcar, y hasta que no le aplican la insulina, no se le controla. Me da mucho miedo que le dé a otra persona de la familia.

G12: Le da a uno tristeza que uno se esfuerza por una paciente, porque este mejor y de un momento a otro le toca salir corriendo con ella al hospital.

H4: Uno no se imagina que las cosas vayan a llegar a tal punto de que el paciente se agrava tanto.

H16: Estamos muy preocupados porque en el último control, tenía el azúcar, el colesterol y los triglicéridos altos, el único que le salió bueno fue el de la tensión, de resto todos los exámenes le salieron malos, y uno bregando a cuidarlo.

J9 "La Diabetes es una enfermedad que no lleva solamente al paciente, lleva a toda una familia de cierta manera a vivir un poco mal."

J11 "Si Dios no se acuerda temprano de ellos se los va es llevando por pedacitos".

Los cuidadores del estudio perciben la enfermedad con grados variables de gravedad. Se ha reportado la percepción de la enfermedad como catalizadora del afrontamiento, si esta se percibe grave, puede motivar una respuesta de estrés positiva o negativa hacia la enfermedad (Lazcano et al., 2008). La percepción de gravedad de la enfermedad suele motivar la búsqueda de aprendizaje por los cuidadores para mejorar el control de la misma. En este sentido, es evidente que los cuidadores asumen con compromiso el desempeño de su rol.

Algunos estudios reportan que la percepción de la enfermedad por la persona afectada genera estrés que puede ser afrontado positivamente con apoyo social familiar. Esto explica la importancia de los procesos de adaptación a la enfermedad, tanto en pacientes como en cuidadores informales para lograr un mejor control metabólico de la enfermedad. En consecuencia, lograr reducción de los episodios de estrés y factores de riesgo en el cuidado de los pacientes crónicos (Vega et al., 2009).

Por otra parte, se ha documentado (Pinto, 2005; Alpuche et al., 2008) los difíciles momentos que deben vivir los cuidadores informales cuando el estrés y el 
agotamiento los embargan, principalmente en estados críticos de la enfermedad. No encuentran mayor apoyo familiar y deben responder a otros por su comportamiento en el cuidado del familiar. Adicionalmente, los cambios emocionales en las personas con DM 2 están bien fundamentados por la literatura científica (Agudelo, 2007). Su condición implica grandes esfuerzos emocionales y físicos, alteración de sentimientos y poca aceptación de su autoimagen, aspectos que inciden en la relación de cuidado entre el paciente y cuidador, si no hay comprensión y manejo adecuado de la situación. Adicionalmente la presencia de un enfermo crónico en la familia suele generar tensiones, cambios en la dinámica familiar y problemas previos pueden hacerse aún más evidentes.

\section{Necesidades de apoyo familiar y social del cuidador para el cuidado de la enfermedad}

El contexto familiar y social en el que vive la persona con diabetes y su cuidador pueden ser obstáculos o dinamizadores en la realización de esfuerzos para el control de la diabetes. De hecho, el apoyo social aportado por la familia, los amigos, los compañeros de trabajo y los profesionales de la salud juega un rol crucial en la capacidad de las personas con diabetes en adoptar y mantener cambios en estilos de vida que les permita controlar la enfermedad (McCloskey et al., 2010). Los cuidadores son quienes perciben el apoyo social disponible como un elemento importante para enfrenar situaciones estresantes relacionadas con la enfermedad (Vega, 2011).

Recientemente, un estudio (Beverly, 2008) examinó las necesidades de cuidado de los pacientes y sus parejas para mejorar el manejo de la diabetes. Los resultados muestran que las parejas tienen la necesidad de información continua, la necesidad de contar con un grupo para compartir esa información, la necesidad de trabajo en equipo dentro de la pareja y de que la pareja se constituya en su propio defensor frente a la diabetes. Esa es la forma de prepararse para retomar el control de sus vidas. De esta manera, tanto el paciente como el cuidador informal requieren de un entorno social enriquecido con diversas experiencias de aprendizaje en donde la acción de equipo juega un rol fundamental.

La categoría de apoyo familiar y social fue reconocida como significativa en la experiencia de cuidado de los cuidadores, debido a que la mayoría expresaron sentimientos de soledad y falta de apoyo, tanto por parte de la familia como del equipo de salud que les proporciona la asistencia. Entre los enunciados significativos relacionados se presentan los siguientes:
A1: En los controles, nunca a ella se le mando con un nutricionista para saber que ella puede comer o no, no puede comer nada con dulce ni tomar nada con dulce, no sabemos los riesgos, los síntomas de alarma, ni nada.

B1: Igual en la comida uno sabe que él se tiene que cuidar, uno es consciente, pero como es según la situación económica uno no va haciendo todo lo que a uno le dicen.

C3: Lo de los medicamentos me gustaría saberlo, cuales medicamentos ella debe utilizar y los cuidados con estos. Lo de la alimentación, porque no sé cómo ayudarla en esa parte; en el ejercicio físico, si es bueno que ella practique algo. Saber si yo estoy en riesgo de sufrir lo mismo que ella, o si mis sobrinas también se pueden enfermar.

D1: Yo no puedo estar todo el tiempo compartiendo con él, porque al igual yo también tengo que hacer mis oficios.

E1: Siempre nos dan los medicamentos, solamente pastillas, no le formulan nada más, no son capaces de formularle una Milanta, solo por ahorrarle plata al gobierno, porque ella sufre de la úlcera también, toca comprársela por aparte, vitaminas y todo eso, el seguro le está sirviendo por una parte, para la diabetes y la tensión, esas dos cosas las está controlando.

N2: Un médico no le explica esto y esto se hace así o esta droga es para esto, únicamente una fórmula, compre y tome.

C2: Mi meta era descansar en mi vejez, ahora tengo 42 años y me toca estar al lado de mi hermana, eso es muy duro.

(I-1) Nunca nos han dado ni siquiera un papel que diga, hay que darle esto de comer... hay que darle esto, nada, lo que sumercedes nos han dicho...me han dicho más ustedes que los propios de allá del Hospital...

M30: Cuando él se vio tan malito, yo llamé a los hermanos y ellos me dijeron que no tenían tiempo para nada. Por allá a veces viene uno, pero eso es... visita de médico, apenas unos minuticos. Nosotros somos los dos solitos.

M31: Yo he bregado primero con mi papá y mi mamá, y ahora con él, y siempre me ha tocado solita, pues uno si quisiera tener a alguien más que ayudara, pero si no hay...que se va a hacer. Le toca a uno valerse por uno mismo. 
En relación al apoyo social por parte de los profesionales de la salud, un estudio realizado en el Reino Unido (Booth, 2008) muestra que dentro de las barreras más significativas para el logro del autocontrol está la necesidad de realizar muchos cambios en muy corto tiempo. Por ejemplo, cocinar y hacer las compras para cumplir con la dieta del enfermo, satisfacer además los gustos del resto de la familia y batallar con la percepción de que las restricciones en la dieta hacen que la alimentación sea una experiencia desagradable. Lo anterior señala la necesidad de que los servicios de salud programen y desarrollen sesiones individualizadas de educación y vinculen al enfermo y al cuidador en grupos de apoyo que los acompañen, y refuercen su habilidad de cuidado. En nuestro medio, el sistema de salud no responde de manera adecuada a las necesidades de conocimientos de los pacientes ni de los cuidadores de las personas con diabetes, con frecuencia. La siguiente expresión de una cuidadora muestra la situación vivida como resultado de la falta de participación de los profesionales de la salud en el diagnóstico y control de la DM 2:

K1 “El se tomó el examen ese del azúcar que llaman glicemia y cuando me llamó me dijo que la tenía en 490 y yo dije bueno, eso qué será...pero pues uno no le pone cuidado porque uno qué va a saber de eso...entonces fue a la consulta pero no le dijeron: vea tiene que tener una dieta, tiene que tener algo, no, pues él siguió comiendo normal, cuando dice el doctor: toca mocharle el pie."

Una intervención de gran relevancia para obtener control metabólico y que frecuentemente requiere de grupos de apoyo es la actividad física realizada junto a amigos, cuidadores, y otros enfermos. Un estudio (Hughes et al, 2007) muestra una experiencia exitosa en la conformación de grupos de apoyo y de fomento de hábitos saludables. Los miembros del grupo de apoyo conformado no solo realizan actividad física, sino que también comparten experiencias de cuidado, disminuyen el estrés y fortalecen las redes sociales para combatir el aislamiento y la depresión, una importante comorbilidad de la diabetes que afecta seriamente las habilidades de autocuidado (Ciechanowski, 2011).

De igual manera, estos grupos de apoyo aumentan la confianza en las habilidades individuales para tomar decisiones y desarrollar experiencias de autodeterminación necesarias en la obtención de conductas de automonitoreo y control de la diabetes (Hutchinson et al, 2008). Adicionalmente, según lo reportado por un estudio realizado en Suecia (Hjelm, 2009), el soporte social ofrecido por los profesionales de la salud debe contener el apoyo informativo y emocional necesario en el momento requerido, de manera que los enfermos y cuidadores cuenten con un tutor permanente que oriente alternativas apropiadas para la adaptación a los nuevos estilos de vida en el control de la enfermedad. Estas estrategias y otras similares pueden ser aplicadas en nuestro medio con el objeto de fortalecer el control de la enfermedad y apoyar al cuidador en su labor de cuidado domiciliario.

Otro aspecto relevante reportado por los cuidadores fue la preocupación por las demandas económicas que la enfermedad impone sobre las familias afectadas. Algunos cuidadores expresan lo siguiente:

M32 "Todo vale (plata) sobre todo lo del transporte y es que me ha tocado ir muchas veces para lo de la tutela (por una droga formulada)."

N37 "Ella toma lo que le formuló la doctora, ese Yogo Yogo pero light, de Alpina, eso también se compra a veces para ella, cuando no se puede un frasco entonces una bolsita."

Las limitaciones económicas reducen las oportunidades de asistir a jornadas de educación, disminuyen las ocasiones de contar con los servicios de especialistas a demanda y generalmente restringe el acceso a información de mayor profundidad. Por lo anterior, los conocimientos del cuidador dependen fundamentalmente de la información suministrada al enfermo durante la consulta con el profesional de la salud, siendo el tiempo de consulta excesivamente limitado.

La evidencia señala que las personas con DM 2 que viven en condiciones de pobreza, tienen mayor probabilidad de discapacidad relacionada con la enfermedad (Schmitz et al, 2009), por lo que se hace necesario individualizar la educación en salud acorde a su nivel educativo, de manera que realmente obtengan los conocimientos necesarios para impedir el desarrollo de complicaciones que generen discapacidad y agudicen el deterioro de su condición socio-económica.

\section{Conclusiones}

La compresión limitada de la enfermedad y sus formas de manejo se traducen en alto riesgo de deterioro temprano y graves complicaciones. Así se muestra la necesidad de acciones educativas específicas que orienten en forma interdisciplinaria el debido cuidado de las personas afectadas por la enfermedad y el autocuidado de los cuidadores informales. 
Percepciones como la incertidumbre, el difícil manejo, la variabilidad de la enfermedad y los cambios del estilo de vida familiar, suelen ser predictores negativos que generan tensiones, alteraciones afectivas y de interrelación entre los miembros de la familia. Muchas veces esto ocasiona crisis frecuentes, pobre adaptación y afrontamiento, así como alteración de las relaciones familiares, principalmente pacientecuidador.

La falta de apoyo familiar, social y del equipo de salud experimentada por los cuidadores frente al manejo de la DM 2, contribuye a desestabilizar las acciones de cuidado y la calidad en la ejecución del rol de cuidador. El acompañamiento familiar y el desarrollo de ajustes de la vida en familia es uno de los principales elementos del control metabólico exitoso y la obtención de capacidades de autocuidado.

Se requiere empoderar al cuidador informal a través de estrategias que fortalezcan el conocimiento, la comprensión de la enfermedad y el acompañamiento permanente a la persona con DM 2, con el fin de mejorar las prácticas de cuidado y lograr intervenciones integrales para el control metabólico. La educación del cuidador informal debe involucrar el conocimiento de la enfermedad, su manejo interdisciplinario, la correcta utilización del servicio de salud y los sitios de apoyo para consultas y asistencias disponibles.

Se evidencia la necesidad de investigar los principios científicos de las prácticas de medicina alternativa, con el fin de validar los cuidados provenientes del saber popular y precisar los beneficios que tienen para el control y manejo de la enfermedad.

Las personas con DM 2 deben conocer sus derechos dentro del sistema de salud y los servicios que les deben ser ofrecidos. Adicionalmente, el ejercicio práctico de los profesionales de enfermería, en sintonía con la protección de derechos de los enfermos, debe orientar a las personas con diabetes y a sus cuidadores informales sobre los servicios a los que deben tener acceso. Eso incluye que el servicio de información responda a las características sociales y de doble vía que se puede ofrecer en los grupos de apoyo organizados por el sistema de salud.

\section{Referencias}

Agudelo ME, Builes MV, García J, Gutiérrez R, Ortiz C, López LM, et al. Percepción de la crítica familiar de pacientes ambulatorios con diabetes Mellitus, trastorno bipolar I y sujetos sanos. Rev Colomb Psiquiat. 2007; 36(2): 213-223.
Alpuche VJ, Ramos B, Rojas ME, Figueroa CG. Validez de la Entrevista de Carga de Zarit en una muestra de cuidadores primarios informales. Psicología y Salud. 2008; 18(2): 237-245.

Alligood MR, Tomey AM. 2011. Modelos y teorías de enfermería. 7ª. ed. Madrid: Elsevier- España.

Astudillo W, Mendinueta C, Granja P. Cómo apoyar al cuidador de un enfermo en el final de la vida. Psicooncología 2008; 5(2-3):359-381.

Beverly E, Wray LA, Miller CK. Practice implications of what couples tell us about type 2 diabetes management. Diabetes Spectrum. 2008; 21(1):39-45

Booth AO, Lowis C, Hunter SJ, McKinley MC. Role of diet and physical activity in the self-management of type 2 diabetes: experiences and opinions of patients and health professionals. Proc Nutr Soc. 2008; 67(67):248

Bustos R, Barajas A, López G, Sánchez E, Palomera R, Islas J. Conocimiento sobre Diabetes Mellitus en pacientes diabéticos tipo 2 tanto urbanos como rurales del occidente de México, Arch Med Fam. 2007; 9(3):147-155.

Carretero S, Garcés J, Ródenas F, San José V. (2006). La sobrecarga de cuidadoras de personas dependientes: análisis y propuestas de intervención psicosocial. Valencia: Tirant lo Blanch. Políticas de Bienestar Social. № 06/2006, pp. 9-99

Castillo JA. El cuidado cultural de enfermería, necesidad y relevancia. Rev Haban Cienc Med. 2008; 7(3):0-0

Ciechanowski P. Diapression: an integrated model for understanding the experience of individuals with co-ocurring diabetes and depression. Clinical Diabetes. 2011; 29(2): 43-49.

Federación Internacional de Diabetes, Atlas Sexta edición [Revisado Noviembre 2013]. URL disponible http:// www.idf.org/diabetesatlas/5e/es/la-carga- mundial?language $=\mathrm{es}$,

González DS. Habilidad del cuidado de los cuidadores familiares de personas en situación crónica por diabetes Mellitus. Avances en Enfermería. 2006; 24(2):28-37

Grau C, Fernández M. Familia y enfermedad crónica pediátrica. Anales Sis San Navarra. 2010; 33(2): 203-212.

Granada, 4 y 5 de octubre de 2007, Index Enferm. 2009; 18(2):100-105.

Sociedad Andaluza de Medicina Familiar y Comunitaria (SAMFyC) 2008. [Revisado Septiembre 2013]. URL Disponible http://www.samfyc.es

Grupo de Cuidado. 2000. Facultad de Enfermería. Cuidado y Práctica de Enfermería, Universidad Nacional de Colombia, $1^{\text {a }}$ edición. Bogotá: Unilibros

Hjelm K, Bertero C. Social support as described by Swedish people diagnosed with type 2 diabetes mellitus. Prim Health Care Res and Dev. 2009; 10(1):26-37.

Hughes M, Porter G, Thomas V. Prime Time Sisters Circle. Evaluating a gender-specific, culturally relevant intervention to decrease major risk factors in mid-life African American women. J Natl Med Assoc. 2007; 99(4):428-438. 
Hutchinson S, Bland A, Kleiber D. 2008. Leisure and stress-coping: implications for therapeutic recreation practice. Therapeutic Recreation Journal. 42, 9-23.

Jofré $\mathrm{V}$, Sanhueza O. Evaluación de la sobrecarga de cuidadoras/es informales. Cienc enferm. 2010; 16(3):111-120.

Lazcano M, Salazar BC, Gómez MV. Validación del instrumento Afrontamiento y proceso de adaptación de Roy en pacientes con Diabetes Mellitus tipo 2. Aquichan. 2008; 8(1):116-125.

Luengo CE, Araneda G, López MA. Factores del cuidador familiar que influyen en el cumplimiento de los cuidados básicos del usuario postrado. Index Enferm. 2010; 19(1):14-18.

Luchetti FF, Zanetti ML, dos Santos MA, Martins TA, Sousa VD, de Sousa CR. Conocimiento y actitudes: componentes para la educación en diabetes. Rev Latino-Am Enfermagem. 2009; 17(4): 468-473.

Martínez MG, Moreno MG, Saucedas PF, Vásquez L, Medina SG, Liñán A. Modelo de manejo de casos en pacientes con diabetes mellitus tipo 2. Invest educ enferm. 2006; 24(1):58-65.

McCloskey J, Flenniken D. Overcoming Cultural Barriers to Diabetes Control: a Qualitative Study of Southwestern New Mexico Hispanics. J Cult Divers. 2010; 17(3):110115.

Medina OM, Díaz AD, Barrientos E, Peña DE. Percepción de la salud y su efecto en el pacientes con diabetes. Av Enferm. 2009; 27(2):13-18

Miyar L, Zanetti ML, Daguano M. El Conocimiento del paciente diabético sobre su enfermedad antes y después de la implementación de un programa de educación en diabetes. Rev. Latino-Am. Enfermagem. 2008; 16(2): 231-237.

Morrison V, Bennett P. Psicología de la salud. Madrid: Pearson Prentice Hall. 2007

Noda JR, Pérez JE, Málaga G, Aphang M. Conocimientos sobre "su enfermedad" en pacientes con Diabetes Mellitus tipo 2 que acuden a hospitales generales. Rev Med Hered. 2008; 19(2):68-72.

Ortiz M, Ortiz E, Gatica A, Gómez D. Factores Psicosociales Asociados a la Adherencia al Tratamiento de la Diabetes Mellitus Tipo 2. Ter Psicol. 2011; 29(1):5-11.

Pérez A, Alonso L, García AJ, Garrote I, González S, Morales JM. Intervención educativa en diabéticos tipo 2. Rev Cubana Med Gen Integr. 2009; 25(4):17-29.

Pinto N, Barrera L, Sánchez B. Reflexiones sobre el cuidado a partir del programa "Cuidando a los Cuidadores". Aquichan. 2005; 5(1):128-137.

Quiceno JM, Vinacia S. Percepción de Enfermedad: Una aproximación a partir de Illness Perception Questionnaire, Psicología desde el Caribe. 2010; 25:56-83.
Rodríguez A, Rodríguez RP. Insuficiencia renal crónica en pacientes con diabetes mellitas de tipo 2 en un área de salud. Medisan. 2009; 13(6).

Romero MN. Investigación, Cuidados enfermeros y Diversidad cultural. : mesa redonda Simposio Internacional sobre Investigación en Enfermería Comunitaria.

Sánchez B. 2000. Fenomenología: Un método de indagación para el cuidado de enfermería. Cuidado y práctica de enfermería. Bogotá: Unibiblos

Sánchez B, Pinto N, Barrera L, Carrillo GM. Programa "Cuidando a Cuidadores Familiares". Medwave. 2008; 8(11):e3663

Sapag JC, Lange I, Campos S, Piette JD. Estrategias innovadoras para el cuidado y el autocuidado de personas con enfermedades crónicas en América Latina. Rev Panam Salud Pública 2010; 27(1):1-9.

Sarmiento M, Castro A, Gómez A, Cifuentes A, Fierro M, Vigoya $\mathrm{T}$. Condiciones socio-demográficas y calidad de vida en pacientes mayores Hospital Departamental de Villavicencio. Orinoquia. 2010; 14(1):75-88

Schmitz N, Nitka D, Gariepy G, Malla A, Wang J, Boyer $\mathrm{R}$, et al. Association between neighborhood-level deprivation and disability in a community sample of people with diabetes. Diabetes care. 2009; 32(11):1998-2004

Shosha GA. Employment of colaizzi's strategy in descriptive phenomenology: a reflection of a researcher. Europ Sci J. $2012 ; 8(27): 30-43$.

Siles J. La naturaleza histórica y dialéctica de los procesos de globalización y su incidencia en la cultura de los cuidados. Index Enferm. 2010; 19(2-3):162-166.

Torres CA, Vargas JE. Vivienda para población desplazada en Colombia: recomendaciones para la política pública y exigibilidad del derecho. Revista INVI. 2009; 24(66):17-86.

Vaquiro S, Stiepovich J. Cuidado informal, un reto asumido por la mujer. Cienc enferm. 2010; 16(2):17-24.

Vega-Angarita OM, González Escobar DS. Apoyo social: elemento clave en el afrontamiento de la enfermedad crónica. Enferm Glob. 2009; 16:0-0.

Vega OM. Percepción del apoyo social funcional en cuidadores familiares de enfermos crónicos: Perception of Functional Social Support. Aquichán. 2011; 11(3):274286.

Venegas BC. Habilidad del cuidador y funcionalidad de la persona cuidada. Aquichán. 2006; 6(1): 137-147.

Zavala M del R, Ríos M del C, García G, Rodríguez CP. Funcionalidad familiar y ansiedad en pacientes adultos con enfermedad crónica. Aquichán. 2009; 9(3): 257270. 